\title{
LoDiM: A Novel Power System State Estimation Method with Dynamic Measurement Selection
}

\author{
Jinghe Zhang, Student Member, IEEE, Greg Welch, Member, IEEE \\ and Gary Bishop
}

\begin{abstract}
For decades, state estimation has been a fundamental aspect of power systems. However for large-scale and widearea interconnected power systems, the required computation makes real-time on-line estimation a major challenge. In this paper we present a new method we call Lower Dimensional Measurement-space (LoDiM) state estimation. LoDiM is based on the Extended Kalman filter-popular because of its efficiency, robustness, and typical accuracy. LoDiM, which can take advantage of modern parallel computation techniques, may be useful for other large-scale, real-time on-line and computationally-intensive state tracking systems beyond the power systems, such as weather forecasting or gas-pipeline state estimation. Although LoDiM is presented in the context of the Kalman filter, the associated measurement selection procedure is not filter-specific, i.e. it can be used with other state estimation methods such as particle and unscented filters. If desired, robust estimation techniques can also be employed to detect and eliminate outlier measurements.
\end{abstract}

Index Terms-Power systems, State Estimation Algorithms, Dynamic Measurement Selection, Power system simulation, Kalman Filter, Parallel Compuatation

\section{INTRODUCTION}

$\mathbf{S}$ TATE estimation plays a basic yet very important role in modern industries. Particularly in the power systems, state estimation generates critical input data for driving other operation functions including real-time security monitoring, load-forecasting, economic despatch, load-frequency control, etc..

During state estimation, typically, a state estimator receives telemetered measurements from a Supervisory Control And Data Acquisition (SCADA) system, and generates a set of state variables that reflect a best estimate of the system conditions. Traditional SCADA measurements are updated every 3-5 seconds; innovative devices such as PMUs [5] can deliver 30 measurements per second. On the contrary, state estimator updates the estimates at every 3-5 minutes. Due to the tremendously increasing size and complexity of the interconnected power networks, tedious computations in state estimation remain obstacles to overcome. Even with modern supercomputers, the massive data processing is still time consuming and memory challenging.

In our previous research [2], a tracking method called single-constraint-at-a-time (SCAAT) was proposed. The idea

This work supported by U.S. DOE grant DE-SC0002271, which is a subcontract from PNNL, PI Zhenyu "Henry" Huang. At DOE, grant managed by Sandy Landsberg, Program Manager for Applied Mathematics Research; Office of Advanced Scientific Computing Research; DOE Office of Science.

The authors are all members of the Department of Computer Science, The University of North Carolina at Chapel Hill, Chapel Hill, NC, 27599-3175 USA e-mail: \{jing2009, welch, gb $\} @$ cs.unc.edu behind the SCAAT approach is to use of a Kalman filter to estimate a globally observable system using only measurements from locally unobservable systems. In each filtering cycle, SCAAT deals with much lower dimensional measurement data. Although SCAAT was first developed for optoelectronic 3D tracking systems, it inspired us to design LoDiM [3], a SCAAT-based algorithm featuring dynamic measurement selection to reduce the computational burden, for state tracking of large-scale systems, e.g. power system, weather forecast system, etc.. In this paper, we present a comprehensive study and proof of LoDiM, including the trade-offs and optimal number of measurements to be selected. Furthermore, our method is expected to significantly facilitate power system operations, and integrate with the powerful tool of hierarchical, distributed estimation as well as parallel computation techniques for more improvement.

The remainder of this paper is organized as follows. Section II introduces the background. In Section III, we present our new approach to perform Kalman filtering upon dynamically selected measurements, in order to reduce the computation requirement dramatically, without sacrificing the tracking quality. Our method is tested on two system models in different sizes in Section IV to demonstrate and analyze the performance. Finally, conclusions and acknowledgement are in Section V and Section VI respectively.

\section{BACKGROUND AND RELATED WORK}

In the modern power systems, measurement devices with higher accuracy and update rate are increasingly deployed, setting new requirements for real-time computations. Significant previous work has attempted to alleviate the computational load problem. One approach is to explore the potential computational power: the authors of [7] used Petri net (PN) theory to achieve the optimum utilization of processors, in a state estimator based on Kalman filter.

Another attempt is to reduce the computational complexity, for example, in [8] the authors proposed a method for systems that have more measurements than states. They described an equivalent state-space system can be used in which the number of measurements equals the number of states. The system model is ideally assumed to be static and linear, and the preparation overhead was ignored in the complexity analysis.

[9] illustrated the measurement selection procedure in Extended Kalman filters. Nevertheless, as stated by the authors, an inherent limitation of the proposed method is that measurement selection is based entirely on the steady-state sensitivity matrix. The shortcomings of this approach include: (1) the 
actual information content of the candidate measurements under typical operating conditions is not considered; (2) the measurement rankings obtained are local and dependent on the steady state chosen as the base case; and (3) dynamic and nonlinear effects are neglected.

Previously in [2], we presented SCAAT, a Kalman-filterbased incremental tracking algorithm using incomplete information. It estimates a globally observable system using only measurements from locally unobservable systems. The underlying principle is that the single observations provide some information about the user's state, and thus can be used to incrementally improve a previous estimate. Based on SCAAT, we proposed LoDiM with more sophisticated measurement selection procedure [3]. Here we present a more comprehensive proof and case study to show that the LoDiM estimation is more stable and reliable, with higher report rates and lower latency than the classic Kalman filter.

\section{Lower Dimensional Measurement-Space State ESTIMATION}

Kalman filtering techniques are extensively used in power system state estimations [13], recent study [6] also applied them in power system bad-data detections. The LoDiM method employs a Kalman filter but incorporates a lower dimensional measurement-space in each cycle. It conducts a special measurement selection procedure, in order to strategically reduce the measurement-space dimension. As a result, it yields much smaller computational load, lower latency, and most importantly, reliable performance. In this section, we will first give a brief introduction to the Kalman filter, then discuss our dynamic measurement selection method and the structure of LoDiM.

\section{A. The Kalman Filter}

The Kalman filter [4] has been used in a wide range of applications from radar tracking to weather forcasting. It is an efficient recursive filter that estimates the state of a process, in a way that minimizes the mean of the squared error.

An assumed linear system can be modeled as a pair of linear stochastic process and measurement equations

$$
\begin{aligned}
x_{k} & =A x_{k-1}+w_{k-1} \\
z_{k} & =H x_{k}+v_{k}
\end{aligned}
$$

where $x \in \mathcal{R}^{n}$ is the state vector, $z \in \mathcal{R}^{m}$ is the measurement vector, $A$ is a $n \times n$ matrix that relates the state at the previous time step $k-1$ to the state at the current step $k$ in the absence of either a driving function or process noise ${ }^{1}$, and $H$ is a $m \times n$ matrix that relates the state to the measurement $z_{k}$. The process noise $w_{k}$ and measurement noise $v_{k}$ are assumed to be mutually independent random variables, spectrally white, and with normal probability distributions

$$
\begin{aligned}
p(w) & \sim N(0, Q) \\
p(v) & \sim N(0, R),
\end{aligned}
$$

\footnotetext{
${ }^{1}$ In practice, the matrix $A$ may change with each time step, but it is assumed to be constant here.
}

where the process noise covariance $Q$ and measurement noise covariance $R$ matrices are often assumed to be constant.

In reality, the process to be estimated and (or) the measurement relationship to the process are often nonlinear. Especially when our objective is to estimate the dynamic states of the power system. A nonlinear system can be modeled using nonlinear stochastic process and measurement equations

$$
\begin{aligned}
x_{k} & =a\left(x_{k-1}, w_{k-1}\right) \\
z_{k} & =h\left(x_{k}, v_{k}\right) .
\end{aligned}
$$

These nonlinear functions can then be linearized about the point of interest $x$ in the state space. To do so one need to compute either or both of the Jacobian matrices

$$
\begin{aligned}
& A=\left.\frac{\partial a(x)}{\partial x}\right|_{x} \\
& H=\left.\frac{\partial h(x)}{\partial x}\right|_{x}
\end{aligned}
$$

where $A$ and $H$ are the partial derivatives of $a$ and $h$ (respectively) with respect to $x$.

The Kalman filter estimates the state by minimizing the $a$ posteriori estimate error covariance, in a recursive predictioncorrection manner [1]. The prediction step is realized by a set of time update equations:

$$
\begin{array}{r}
\text { Prediction: } \\
\left\{\begin{array}{l}
\hat{x}_{k}^{-}=A \hat{x}_{k-1} \\
P_{k}^{-}=A P_{k-1} A^{T}+Q
\end{array}\right.
\end{array}
$$

The time update equations are responsible for projecting forward (in time) the previous state $x_{k-1}$ and error covariance estimates $P_{k-1}$ to obtain the a priori estimates for the next time step $k$.

The correction step is carried out by a set of measurement update equations:

$$
\left\{\begin{array}{l}
\text { Correction: } \\
K_{k}=P_{k}^{-} H^{T}\left(H P_{k}^{-} H^{T}+R\right)^{-1} \\
\hat{x}_{k}=\hat{x}_{k}^{-}+K_{k}\left(z_{k}-H \hat{x}_{k}^{-}\right) \\
P_{k}=\left(I-K_{k} H\right) P_{k}^{-}
\end{array}\right.
$$

where $K$ is a $n \times m$ matrix called the Kalman gain matrix, $z_{k}$ is the actual measurement at time step $k, H \hat{x}_{k}^{-}$is the predicted measurement at time step $k$, and $\left(z_{k}-H \hat{x}_{k}^{-}\right)$is called the residual.

$K$ reflects how we trust the actual measurement $z_{k}$ versus the predicted measurement $H \hat{x}_{k}^{-}$. From its expression, one can tell that larger values of $R$ place more weight on the predicted value while smaller values of $R$ place more weight on the measured values. The measurement update equations are responsible for the feedback, i.e. for incorporating a new measurement into the a priori estimate to obtain an improved a posteriori estimate.

\section{B. Principle of Design}

As one would expect, performing dynamic state estimation with $\mathrm{KF} / \mathrm{EKF}$ (any filter) is a rather computationally intensive process. For small systems, the computation could be fast enough for real time control applications. However there are 
three factors that increase the computational effort: the size of the system, the complexity of model components, and the number of measurements to be processed. In the "Correction" phase corresponding to equation (10), we have noticed the expensive cost of calculating the Kalman gain $K_{k}$. Because it involves the inversion of a $m \times m$ matrix $\left(H P_{k}^{-} H^{T}+R\right)$, with complexity of $O\left(\mathrm{~m}^{3}\right)$. This makes the computation intractable when the number of measurements $m$ is too large, which unfortunately, is true for the modern power systems.

On the other hand, if we can reduce the measurementspace dimension, i.e., use only a subset of the available measurements to update (perhaps a subset of) the states during each Kalman filter cycle, the computation cost could be reduced dramatically while still maintaining observability over time and improving accuracy per the SCAAT approach [2]. Specifically we propose using a subset of $m_{\sigma}$ measurements, where $1 \leqslant m_{\sigma} \ll m$. The question is which subset of $m_{\sigma}$ measurements to use in each cycle. Previous work suggests pre-determined measurement subsets, however the dynamic nature of today's power systems requires more flexibility. Performing a principal component analysis (PCA) on the error covariance $P$, we can determine the subset of the state space to be updated most urgently (e.g. with larger estimation uncertainty than others).

Because covariance matrices are always symmetric and positive semidefinite, they have several important properties. Before the measurement update ("Correction" phase (10)) begins, let us consider the PCA of the a priori error covariance matrix $P^{-}=U \cdot D \cdot U^{T}$ :

1) There exists an orthonormal basis $U\left(U U^{T}=U^{T} U=\right.$ $I$, where $I$ is the identity matrix), whose columns are the eigenvectors of $P^{-}$, such that the error covariance matrix expressed in this basis is diagonal. The axes of this new basis are called the Principal Components of $P^{-}$.

2) As the off-diagonal elements of this new diagonal covariance matrix $D$ are zeros, the new variables defined by this new basis (the projections of the a priori estimate error $e^{-}=x-\hat{x}^{-}$on the Principal Components) are uncorrelated.

3) The diagonal elements of this new matrix $D$ are the eigenvalues of $P^{-}$. So the variances of the projections of error $e^{-}$on the Principal Components are equal to the corresponding eigenvalues of $P^{-}$.

4) The eigenvalues in $D$ are ordered decreasingly. The $m$ th eigenvalue corresponds to the $m$ th eigenvector.

The principal components that correspond to the largest elements of $D$, indicate the axes in the state space that have the largest estimation uncertainties. We prefer to target at these uncertainties first, thus we need to find the set of measurements that can be used to reduce these uncertainties most efficiently. For example, in a 3D tracking application example, if we use several cameras to estimate the location of a certain object, and we noticed the uncertainty is growing rapidly in one direction, then in the next cycle of filtering we would ideally use a camera which is looking in an orthogonal direction.

\section{Measurement Selection Procedure}

Similar to SCAAT, LoDiM also constrains the unknowns over time and refines the estimation continually, rather than waiting for a complete collection of observations to form. Nonetheless, it is the measurement selection that differentiates LoDiM from SCAAT. In LoDiM, we select the measurements that benefit our estimate the most (i.e. reduce estimation uncertainty most effectively) during each iteration.

After the "Prediction" phase (9) of each Kalman filter iteration, we have the $n \times n$ a priori error covariance matrix

$$
P^{-}=U \cdot D \cdot U^{T}
$$

where $D$ is the diagonal matrix consisting of the eigenvalues of $P^{-}$in decreasing order, and $U$ is the orthonormal basis whose columns are the corresponding eigenvectors. Notice that the full PCA can also be a quite time consuming process, especially if the state space is large. For this reason, in this paper we only investigate the first eigenvector $u_{1}$ in $U$, which represents the directions that we are most uncertain about in the state space. The largest eigenvalue and the corresponding eigenvector $u_{1}$ can be conveniently obtained by existing algorithms such as the power method [11].

Now consider the measurements. We can rewrite the measurement equation as

$$
z=H x+v=H U U^{T} x+v=(H U) x^{\prime}+v=H^{\prime} x^{\prime}+v
$$

Where $x^{\prime}=U^{T} x$ is the new state vector defined by the new basis $U, H^{\prime}$ is the corresponding $m \times n$ new measurement Jacobian matrix, and $v$ is the unchanged measurement noise vector with $p(v) \sim N(0, R)$. Here we assume $R$ to be a $m \times m$ diagonal covariance matrix, i.e. the measurement noises are uncorrelated.

Because the basis $U$ are composed of unit vectors, $H_{i j}^{\prime}$ can also be considered as the magnitude of the projection (i.e. the scalar projection) of the $i$ th measurement direction vector in the direction of the $j$ th basis in $U$. Notice that we now have

$$
H u_{1}=\left[\begin{array}{lll}
H_{11}^{\prime} & H_{21}^{\prime} & H_{31}^{\prime} \ldots H_{m 1}^{\prime}
\end{array}\right]^{T}
$$

Intuitively, for the same basis, say $u_{1}$, the larger $H_{i 1}^{\prime}$ is, the better the corresponding $i$ th measurement could reduce the uncertainty in this basis direction. However we should also keep in mind that different measurements have different amounts of noise. Thus for $u_{1}$ we create a "ranking" vector $r_{1}$ from $H^{\prime}$ and $R$ using the following adjustment:

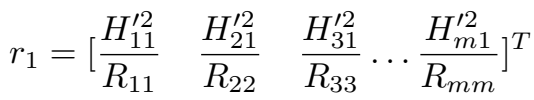

where $R$ is the measurement noise covariance matrix. The $m \times 1$ vector $r_{1}$ evaluates the uncertainty calibration abilities of each measurement regarding the most significant uncertainty component, scaled by the corresponding measurement noise level. Now we are going to prove the following:

Lemma 1: The larger $\frac{H_{i 1}^{\prime 2}}{R_{i i}}$ is, the more effectively the $i$ th measurement can reduce the uncertainty along direction $u_{1}$. 
Proof: It has been shown in [10] that the error covariance update in the "Correction" phase (10)

$$
\begin{aligned}
P & =(I-K H) P^{-} \\
& =\left(I-P^{-} H^{T}\left(H P^{-} H^{T}+R\right)^{-1} H\right) P^{-} \\
& =P^{-}-P^{-} H^{T}\left(H P^{-} H^{T}+R\right)^{-1} H P^{-}
\end{aligned}
$$

is equivalent to

$$
P^{-1}=\left(P^{-}\right)^{-1}+H^{T} R^{-1} H
$$

The inverse of the error covariance, $P^{-1}$, is often called the information matrix. According to (11), we have

$$
\begin{aligned}
P^{-1} & =\left(U D U^{T}\right)^{-1}+H^{T} R^{-1} H \\
& =U^{-T} D^{-1} U^{-1}+U^{-T} U^{T} H^{T} R^{-1} H U U^{-1} \\
& =U^{-T}\left[D^{-1}+(H U)^{T} R^{-1}(H U)\right] U^{-1} \\
& =U^{-T}\left(D^{-1}+H^{\prime T} R^{-1} H^{\prime}\right) U^{-1}
\end{aligned}
$$

Let us denote the matrix $\left(D^{-1}+H^{\prime T} R^{-1} H^{\prime}\right)$ in equation (17) by $\Sigma$, then $\Sigma_{11}$ is the "information" we now have regarding the new state variable in the direction $u_{1}$, which we were most uncertain about. This information value is increased/improved from $D_{11}^{-1}$ to $\Sigma_{11}$ by

$$
\left(H u_{1}\right)^{T} R^{-1}\left(H u_{1}\right)=\sum_{i=1}^{m} \frac{H_{i 1}^{\prime 2}}{R_{i i}}
$$

So if we are only willing to incorporate $m_{\sigma}$ measurements (instead of the full $m$ measurements) into the measurement update equation, the ones with the largest $\frac{H_{i 1}^{\prime 2}}{R_{i i}}$ values would be our choice.

We are able to easily locate the $m_{\sigma}$ measurements with the largest values among these $m$ elements in vector $r_{1}$ (14). Thus when our budget of computation time and memory space is tight, we could use only $m_{\sigma}$ measurements in the next step but still achieve stable estimation results. Using this approach to reduce state estimation uncertainty is similar to fighting the Hydra in Greek mythology: if we are not able to destroy all the "heads" at once, at least we can aim at and cut off the most threatening "head" during each round.

\section{LoDiM Architecture}

For computational efficiency in large-scale Kalman-filterbased state estimations, we propose a better realization for our new method, LoDiM, consisting of algorithm parallelization and parallel implementation.

To generalize our approach, let us consider a nonlinear system described by equations (5) and (6), with a huge measurement space. LoDiM has its main state estimation process, which is similar to SCAAT algorithm, running on the foreground:

1) Compute the time $\Delta_{t}$ since the previous estimate.

2) Predict the state and error covariance. Share the predicted error covariance $P^{-}$with the background process.

$$
\left\{\begin{array}{l}
\hat{x}^{-}=a_{\Delta_{t}}\left(\hat{x}_{t-\Delta_{t}}, 0\right) \\
P^{-}=A_{\Delta_{t}} P_{t-\Delta_{t}} A_{\Delta_{t}}^{T}+Q_{\Delta_{t}}
\end{array}\right.
$$

3) If this is the first cycle, choose $m_{\sigma}$ measurements (which is a much smaller measurement subset) randomly; otherwise, choose the $m_{\sigma}$ measurements nominated by the background process. Predict the measurement and compute the corresponding Jacobian.

$$
\left\{\begin{array}{l}
\hat{z}=h_{\sigma}\left(\hat{x}_{t}^{-}, 0\right) \\
H=H_{\sigma}\left(\hat{x}_{t}^{-}, 0\right)
\end{array}\right.
$$

4) Compute the Kalman gain.

$$
K=P^{-} H^{T}\left(H P_{k}^{-} H^{T}+R_{\sigma, t}\right)^{-1}
$$

5) Correct the predicted state estimate and error covariance from (19) using the actual sensor measurement $z_{\sigma, t}$.

$$
\left\{\begin{array}{l}
\hat{x}_{t}=\hat{x}^{-}+K\left(z_{\sigma, t}-\hat{z}\right) \\
P_{t}=(I-K H) P^{-}
\end{array}\right.
$$

While at the same time, LoDiM has its auxiliary measurement selection process as described in subsection III-C, running on the background:

1) Compute the principal component $u_{1}$ of the error covariance $P^{-}$predicted on the foreground.

2) Compute $H u_{1}$ according to (13) and the ranking vector $r_{1}$ according to (14).

$$
r_{1}=\left(H u_{1}\right) \cdot *\left(H u_{1}\right) \cdot / \operatorname{diag}(R)
$$

where .* and ./ denote the element-by-element operations.

3) Select the $m_{\sigma}$ measurements that correspond to the $m_{\sigma}$ largest elements in $r_{1}$, to be used by the foreground process.

Overall, the whole LoDiM algorithm process can be expressed by the flow chart in Figure 1.
KF/EKF (foreground)

Cycle 1

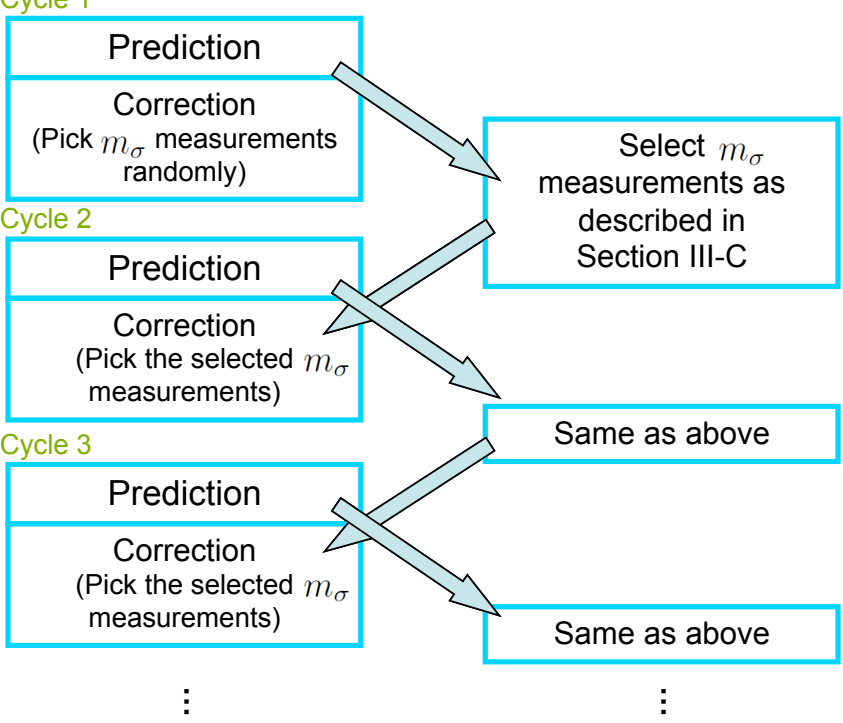

Fig. 1. The LoDiM algorithm flow chart. 


\section{Simulation Results}

\section{A. Evaluation}

In this subsection, we investigate the performance of the proposed LoDiM state estimation using two different power system models: a smaller 3-generator 9-bus system, and a larger 16-generator 68-bus system representing the New England/New York interconnected system [15]. We simulate a large disturbance emergency event: a three-phase fault happens at $t=1$, and is then cleared in 0.15 second.

The state variables (voltage magnitude and phase angle at every bus) are recorded as the "true" system states. For the smaller system, there are 36 simulated Phasor Measurement Unit (PMU) measurements, combined with random noise. PMUs provide both voltage phasor and current phasor measurements, at a higher frequency and accuracy level [5]. We will visualize the impact of measurement selections via the state estimation at bus 2 , a generator bus.

In contrast to LoDiM with $m_{\sigma}=6$, we examine the performance of reduced measurement-space state estimation with a naive approach: the same number of the measurements are chosen randomly during each cycle. Fig. 2 provides a close look at bus 2 voltage magnitude tracking within a 1 second time period from $t=2$ to $t=3$ : while the true state is still plotted in black, the magenta dash-dot line and the blue dashed line represent the estimated states using random measurement selection and LoDiM respectively.

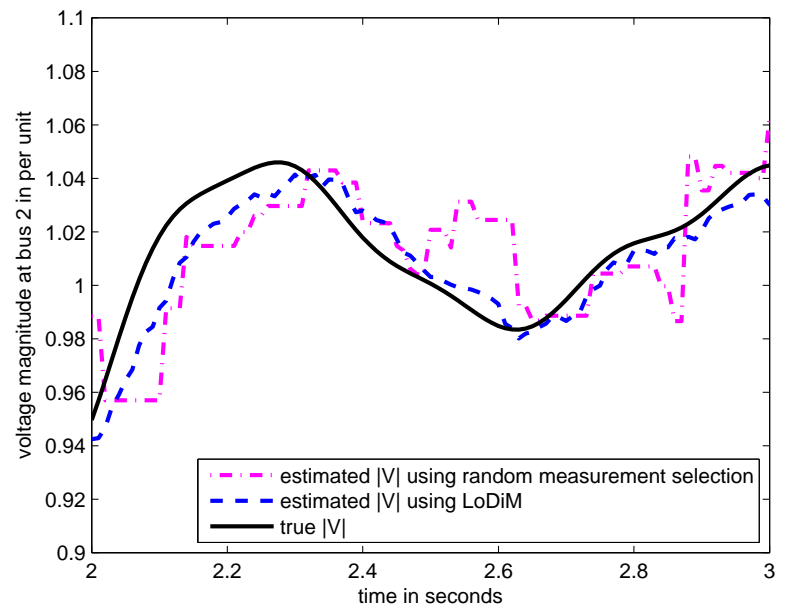

Fig. 2. The performance comparison of bus 2 voltage magnitude estimation using randomly chosen measurements and LoDiM

Similarly in the larger system, we simulated 272 PMU measurements and compare the estimations at bus 26, a load bus, with $m_{\sigma}=70$ (Fig. 3). Both figures demonstrate that the measurement selection procedure significantly improves the state tracking performance.

\section{B. Optimal Number of Selected Measurements}

$m_{\sigma}$, the number of measurements to be selected in each cycle, affects the estimation results. Fig. 4 presents the zoomin bus 2 voltage magnitude tracking results for the smaller system, from $t=2$ to $t=3$. The true state is recorded

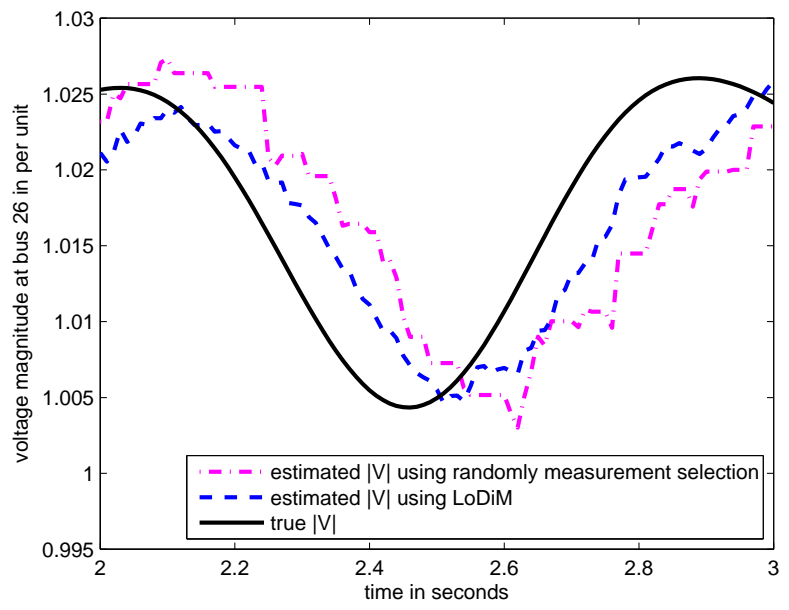

Fig. 3. The performance comparison of bus 26 voltage magnitude estimation using randomly chosen measurements and LoDiM

by black solid line. Our LoDiM state estimation method uses dynamically selected measurements during each cycle as described in section III-C. The blue dash-dash line plots the well-estimated state when $m_{\sigma}=6$. If $m_{\sigma}$ is too small, e.g. $m_{\sigma}=2$, the estimated state resembles a step function as shown in green dash-dot line. This is because very little information is integrated in each cycle, while the speed-up of the cycle period is not significant enough to capture the state variation. On the other hand, the red dotted line plots the estimated state using conventional Kalman filter, where $m_{\sigma}$ is too large that the entire measurement set is used as input, resulting in lower estimation rate.

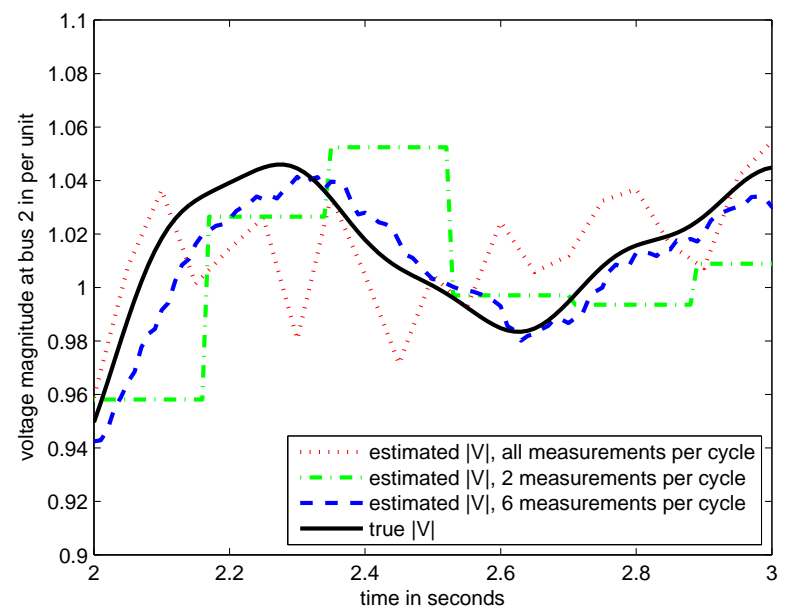

Fig. 4. The performance comparison of bus 2 voltage magnitude estimation with different $m_{\sigma}$ values

Similar estimation behaviors are observed in the larger system, as shown in Fig. 5.

Depicted above, the LoDiM performance comparison with different $m_{\sigma}$ values reflects an interesting trade-off between gained information and processing time. This observation leads us to believe that there exists a sweet spot to achieve 


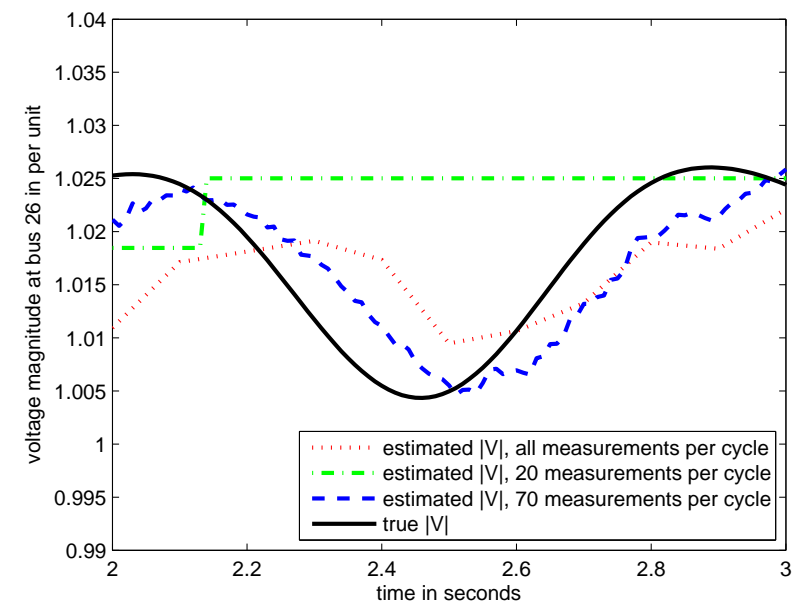

Fig. 5. The performance comparison of bus 26 voltage magnitude estimation with different $m_{\sigma}$ values

optimal performance. To confirm our supposition, we test LoDiM with all possible $m_{\sigma}$ 's, i.e. from $m_{\sigma}=1$ to full measurement-space on both power system models.

We evaluate the performances based on a fair competition. The mean state estimation error (in percentage) of all buses using different amount of measurements (also in percentage) are plotted for these two test systems respectively in Fig. 6 and 7. As a result, the optimal amount of measurement to be selected during each cycle is $77 \%$ for the small system, $26 \%$ for the large system.

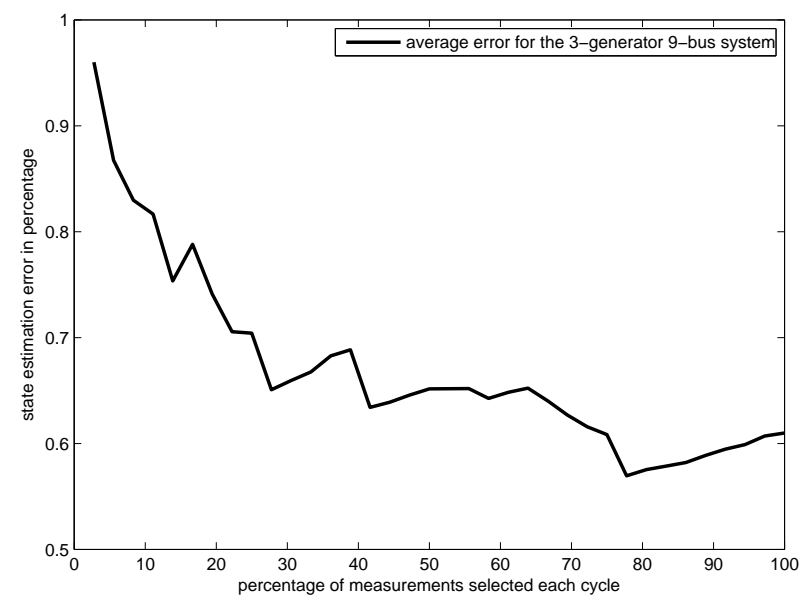

Fig. 6. LoDiM performance with different amount of selected measurements in the 3-generator 9-bus system.

\section{Conclusions}

We presented LoDiM, a novel state estimation algorithm. In each estimation cycle, compared to traditional KF/EKF state estimation methods which handle the entire measurementspace, it deals with a lower dimensional measurement-space. The smaller measurement-space incorporate less information each cycle, but has higher reporting rates. We can analyze and adjust the trade-off to achieve optimal performance.

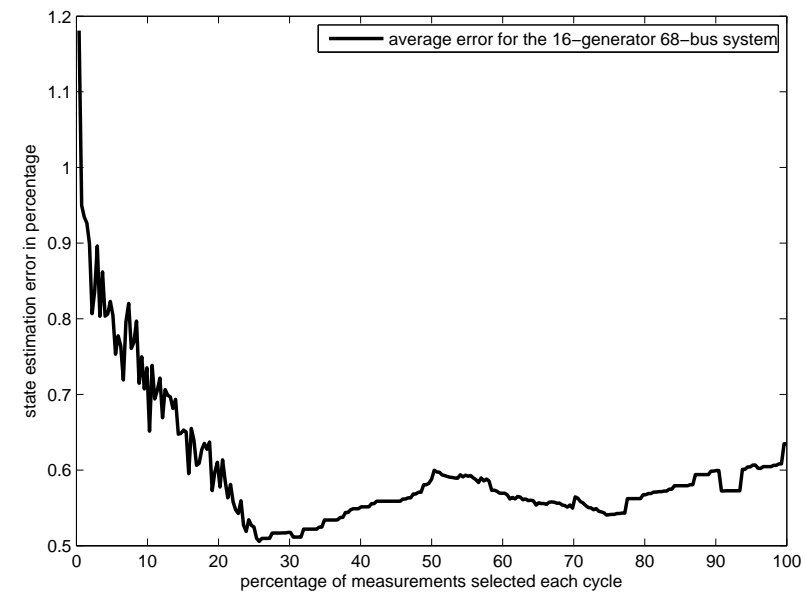

Fig. 7. LoDiM performance with different amount of selected measurements in the 16-generator 68-bus system.

LoDiM features a dynamic measurement selection procedure: a measurement subset which benefits the estimation the most is dynamically chosen for each cycle. The simulation results illustrate a promising future of LoDiM in large-scale dynamic state estimations, for the power systems and beyond. Moreover, it can be parallelized for further optimizations.

Although LoDiM is presented in the context of the Kalman filter-popular because of its efficiency, robustness, and typical accuracy [12] [13] [6], the associated measurement selection procedure is not filter-specific, i.e. it can be used with other state estimation methods such as particle and unscented filters. If desired, robust estimation techniques can also be employed to detect and eliminate outlier measurements [14].

\section{ACKNOWLEDGEMENTS}

At the Pacific Northwest National Laboratory we acknowledge Zhenyu Huang, Ning Zhou, Pengwei Du, and Ruisheng Diao for helpful background discussions. This work is supported by U.S. Department of Energy (grant DE-SC0002271 "Advanced Kalman Filter for Real-Time Responsiveness in Complex Systems," PIs Zhenyu Huang at PNNL and Greg Welch at UNC), Sandy Landsberg, Program Manager for Applied Mathematics Research; Office of Advanced Scientific Computing Research; DOE Office of Science. This work was also supported in part by the Office of Naval Research (award N00014-09-1-0813, “3D Display and Capture of Humans for Live-Virtual Training,” Dr. Roy Stripling, Program Manager.

\section{REFERENCES}

[1] Greg Welch, and Gary Bishop “An introduction to the Kalman Filter". TR 95-041, Department of Computer Science, University of North Carolina at Chapel Hill, April 2004.

[2] Greg Welch and Gary Bishop, "SCAAT: Incremental Tracking with Incomplete Information”. Computer Graphics. T. Whitted. Los Angeles, CA, USA (August 3 - 8), ACM Press, Addison-Wesley: 333-344. 1997

[3] Jinghe Zhang, Greg Welch, and Gary Bishop, "Power System State Estimation with Dynamic Optimal Measurement Selection”. To appear in Proceedings of 2011 IEEE Symposium on Com- putational Intelligence Applications in Smart Grid, (Paris, France), 11C15 April 2011

[4] R. E. Kalman "A new approach to linear filtering and prediction problems". Transaction of the ASME Journal of Basic Engineering, 82(Series D):35-45, 1960. 
[5] A. Phadke, J. Thorp, and M. Adamiak, "A new measurement technique for tracking voltage phasors, local system frequency, and rate of change of frequency" Power Apparatus and Systems, IEEE Transactions on, vol. PAS-102, no. 5, pp. 1025-1038, may 1983.

[6] Chien-Hung Huang, Kuang-Kong Shih, Chien-Hsing Lee and Yaw-Juen Wang "Application of Kalman Filter to Bad-Data Detection in Power System ". InTech 2010

[7] Anant Oonsivilai and Kenedy A. Greyson "Optimization of Distributed Processors for Power System: Kalman Filters using Petri Net". World Academy of Science, Engineering and Technology 532009

[8] M.J. Goris, D.A. Gray and I.M.Y. Mareels "Reducing the computational load of a Kalman filter". Electronic Letters Vol. 33 No. 181539 -1541, 28th August 1997

[9] Shoujun Bian, Michael A. Henson "Measurement selection for on-line estimation of nonlinearwave models for high purity distillation columns". Chemical Engineering Science 61 (2006) 3210-3222

[10] Frank L. Lewis "Optimal Estimation with an Introduction to Stochastic control theory". John Wiley and Sons, Inc. 1986

[11] Golub, G. H. and Van Loan, C. F "Matrix Computations". Johns Hopkins Univ. Press, Baltimore, MD, 1989.

[12] Zhenyu Huang, Kevin Schneider, and Jarek Nieplocha "Feasibility Studies of Applying Kalman Filter Techniques to Power System Dynamic State Estimation" The 8th International Power Engineering Conference IPEC2007 3-6 December 2007, Singapore

[13] Amit Jain, Shivakumar N. R. "Power System Tracking and Dynamic State Estimation" IEEE PES Power Systems Conference Exposition (PSCE) 2009

[14] Jo-Anne Ting, Evangelos Theodorou and Stefan Schaal "Learning an Outlier-Robust Kalman Filter" Lecture Notes in Computer Science, 2007, Volume 4701/2007, 748-756

[15] Joe H. Chow and Kwok W. Cheung "A Toolbox for Power System Dynamics and Control Engineering Education and Research" Transactions on Power Systems, Vol. 7, No. 4, November 1992

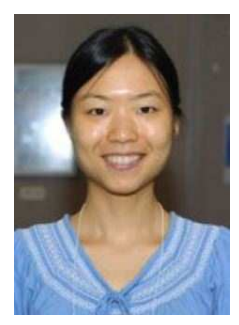

Jinghe Zhang is a graduate student in computer science at the University of North Carolina (UNC) at Chapel Hill. Her current research interests include optimal sensor placement for the power grid, and large scale estimation in general. Zhang has a B.S. from the University of Science and Technology of China (2006), and an M.S. from the University of Idaho (2008), both in mathematics.

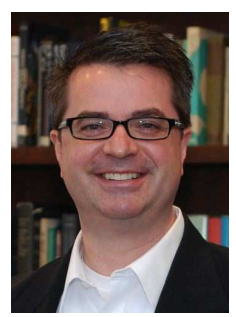

Greg Welch is a research associate professor of computer science at the University of North Carolina (UNC) at Chapel Hill. His primary research areas include stochastic estimation, virtual and augmented reality, human tracking systems, and 3D telepresence. Welch has a B.S.E.T. from Purdue University (1986), and a Ph.D. in computer science from UNCChapel Hill (1995). He is a member of the IEEE Computer Society and the ACM.

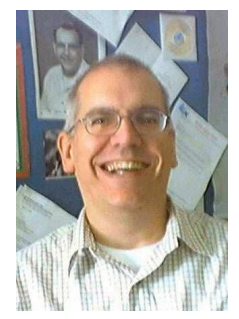

Gary Bishop is a professor of computer science at the University of North Carolina (UNC) at Chapel Hill. His current research interests include applications of computer technology to address the needs of people with disabilities, and systems for manmachine interaction. Bishop has a B.S.E.E.T. from Southern Technical Institute (1976) and a Ph.D. in computer science from UNC-Chapel Hill (1984). 